\title{
Finger Displacement Sensing: FEM Simulation and Modelling of a Customizable Three-Layer Electrode Design
}

\author{
$\mathrm{Nan} \mathrm{Hu}$ \\ Electronics and Computer Science Department \\ University of Southampton \\ Southampton, UK \\ nh1u16@soton.ac.uk
}

\author{
Paul H Chappell \\ Electronics and Computer Science Department \\ University of Southampton \\ Southampton, UK \\ phc@ecs.soton.ac.uk
}

\author{
Nick R Harris \\ Electronics and Computer Science Department \\ University of Southampton \\ Southampton, UK \\ nrh@soton.ac.uk
}

\begin{abstract}
Home based or tele technological systems and smart devices have provided alternative delivery forms to promote hand rehabilitation. As a step towards the targeted system, a finite element method (FEM) simulation based on MGC3130 three-layer electrode design in Comsol®, and a nonlinear regression analysis using Matlab ${ }^{\circledR}$ were carried out. Concerning different combinations of fingers' movement and the symmetrical structure of the simulation model, nine cases in total are simulated. In each case, there are ten testing points, ranging from $0 \mathrm{~mm}$ to $30 \mathrm{~mm}$, to explore the inherent relationship between the distance changed from finger motion and the voltage signals detected in the receive electrodes. The results in both the original electrode design and the modified electrodes design agree with the quasi-static electrical near field theory and the symmetrical structure of the three-layer electrodes. Based on the simulation result, the functional relationship of the data was also investigated. The nonlinear equation, describing the performance of the electrode layers, fits well in both electrode designs, which implies a clear inverse relation between the changed distance and the detected voltage signals. The equation also reflects the sensitive and finite features of the design, which helps to guide and optimize the practical design of the electrodes in the future investigations.
\end{abstract}

Keywords-finger movement detection; Comsol ${ }^{\circledR}$ simulation; capacitive sensor; electrode design; hand rehabilitation

\section{INTRODUCTION}

The emergence of some technological systems and smart devices that realize home based or tele-hand rehabilitation has exposed alternative delivery forms to promote patients' hand recovery from some common physiological conditions [1-5]. It includes tremor, Parkinson's disease, dystonia and initial flaccidity (reduced muscle tone) caused by stroke, which may lead to spasticity (increased muscle tone), excessive reflexes, and obligatory synergies [6]. Investigations into home based or tele hand rehabilitation techniques are especially important, given the noticeable economic burden on patients and the whole society, as well as the great reliance on healthcare facilities and professionals. The major barrier to this adoption, however, is that patients cannot always undertake the rehabilitation sessions properly. For instance, without continuous supervision from a therapist, they might get bored and give up easily, become lazy and start to not correctly follow the procedures, or even misinterpret the therapy and result the potential for injury. Therefore, new technological approaches that realize home based rehabilitation in a low-cost smart equipment should be investigated [5].

Sensing technologies that could be used for the finger motion detection mainly include inertial sensor based technology, Electromyography (EMG) [7, 8], vision based systems [9], magnetic sensor based technology, depth based technology [10-13], glove based systems [10, 13-15], robotaided technology [14] as well as capacitive sensing technology $[5,15,16]$. Capacitive sensors provide the possibility of noncontact detection, which means it is capable of calculating the proximity of an object without a sensing element attached to the object. Further to this, unlike vision-based systems or EMG based technology, it works without a large overhead in signal processing. Moreover, the sensitivity of the sensing decreases quickly when the distance between the sensor and human body increases [16], making it particularly sensitive to the small and easy movements, like the movement of fingers.

After comparing the strengths and weakness of products readily available in the market for hand motion tracking, the MGC3130 motion sensor was chosen as a low cost and reliable off-the-shelf option, given the economic reasons and time restrictions $[5,17]$. It uses the principle of electrical near field sensing for advanced proximity detection to provide gesture and positional data of a human hand in real time. It is a robust and advanced solution for gesture controlled systems in embedded applications [18].

Application of the MGC3130 module includes electrode design and simulation, module integration, and module parameterization. In this paper, a finite element method (FEM) 
simulation based on a customizable three-layer electrode design in Comsol ${ }^{\circledR}$ Multiphysics, and a nonlinear regression analysis using Matlab ${ }^{\circledR}$ were carried out to guide the practical design of the electrodes [20].

\section{Simulation Model}

\section{A. Three-Layer Electrode Stack-Up Design}

The Comsol ${ }^{\circledR}$ simulation model of electrode stack-up (three-layer) design consists of receive electrodes $(\mathrm{Rx})$, a transmit electrode (Tx), a ground electrode (GND) and isolation layers $[5,20]$, as presented in Fig.1 (a). This twodimensional simulation model is customizable by size parameter setting of each component for broader application. An exemplar receptacle model is also shown in Fig. 1. The four fingers of the human hand are modelled as conductive cylinders placed on the top layer. The hand is pronated with the fingers extended and the thumb adducted so that the hand adopts a flat posture. Each finger can be extended from the flat posture but not into hyperextension. Between Top layer and isolation layer1, four Rx electrodes are placed underneath four fingers (I: index, M: middle, R: ring; L: little) accordingly to track their motion. The Tx electrode and the GND electrode are placed between isolation layer1 and isolation layer2, isolation layer2 and bottom layer respectively.

In the Comsol ${ }^{\circledR}$ simulation package, all the electrodes are set to use the default material 'copper', while the 'Acrylic plastic' is chosen as the material of the isolation layers with necessary parameter settings (relative permittivity and density). To simplify the simulation model of a human finger without losing its important electrical physical characteristics, 'water' was chosen to represent the soft and hard tissues of a finger. 3.3 V A.C is applied to Tx electrode whereas both the GND electrode and the fingers are set to the ground potential.

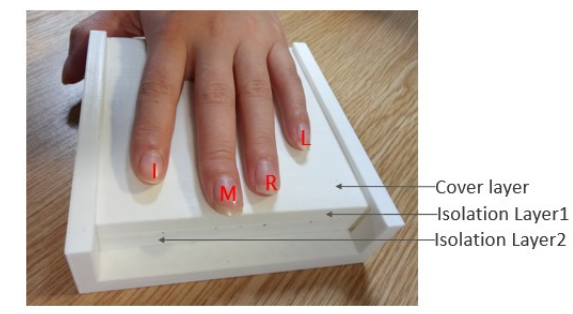

(a)

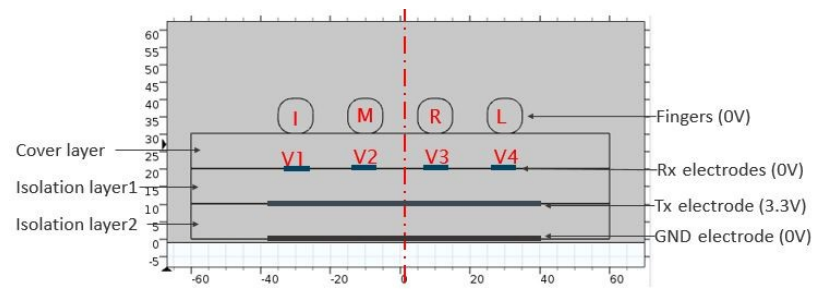

(b)

Fig. 1 Electrode stack-up design (I: index; M: middle; R: ring; L: little): (a) Experimental hand receptacle; (b) $\mathrm{Comsol}^{\circledR}$ simulation model

\section{B. Fingers Movements}

Concerning different combinations of fingers' movement and the symmetrical structure of the simulation model, nine cases in total are simulated to explore all possible combinations of finger movement. As presented in Table 1, there are two cases (case 1 and case 2 only, as case 1-2 and case 2-2 are regarded as the symmetrical cases which will end up with similar outcomes) if one finger moves; four cases (case3, case4, case5 and case6) if two fingers move together; two cases (case 7 and case8) if three fingers move together and one case (case9) if all four fingers move together.

\section{ELECTRIC FIELD DISTRIBUTION VARYING WITH FINGER MOTION}

The MGC3130 motion sensor is based on the quasi-static electrical near field theory for advanced proximity sensing. When conductive objects, such as a person's hand or fingers, intrude the electrical field, the field lines are drawn to the hand or shunted to the ground and the three dimensional electric field decreases locally. This minuscule change then causes a compression of the equipotential lines and shifts the $\mathrm{Rx}$ signal levels to a lower potential that is measured [18].

As shown in Fig. 2 and Fig. 3, case2-2 (ring finger moves: 0010) is chosen as the exemplar finger motion for simulation in this paper to show the influence of an earth-grounded body to the electric field distribution. Underneath each finger, four $\mathrm{Rx}$ electrodes are used to detect the E-field variations at different positions to measure the origin of the electric field distortion from the varying signals received. A variable ' $d$ ', which is the distance between the under surface of a finger and the top of the cover layer, was used to represent the approaching and departure of the third finger(R). In other words, it is a height parameter to record the changes in the vertical coordinate, as shown in Fig. 2 as the red vertical lines below the ring finger. Ten values of the height parameter were selected to repeat in each case. The distances range from $0 \mathrm{~mm}, 2 \mathrm{~mm}, 4 \mathrm{~mm}, 6 \mathrm{~mm}$, $8 \mathrm{~mm}, 10 \mathrm{~mm}, 15 \mathrm{~mm}, 20 \mathrm{~mm}, 25 \mathrm{~mm}$ to $30 \mathrm{~mm}$.

As the 'ring finger' moves away from the model surface, the voltages of the $\mathrm{Rx}$ electrodes (Vx: V1, V2, V3, V4) increase accordingly as the input signal to reflect this motion. The voltages of electrodes $(\mathrm{Vx})$, corresponding to the varied distance, were calculated in Comsol ${ }^{\circledR}$ using point evaluation, as presented in Fig. 3(a). Similarly, the plots of case 2 (Middle finger moves: 0100) were produced, as shown in Fig. 3 (b).

TABLE 1 FINGERS MOVEMENTS DISCUSSION IN SIMULATION

\begin{tabular}{|c|c|c|c|c|c|c|c|c|c|c|}
\hline \multirow[t]{2}{*}{ Category } & \multicolumn{5}{|c|}{ Finger Combinations } & \multicolumn{5}{|c|}{$\begin{array}{c}\text { Symmetrical Finger } \\
\text { Combinations }\end{array}$} \\
\hline & Case & $I$ & $M$ & $R$ & $L$ & Case & $I$ & $M$ & $R$ & $L$ \\
\hline \multirow{2}{*}{$\begin{array}{l}\text { One finger } \\
\text { moves }\end{array}$} & 1 & 1 & 0 & 0 & 0 & $1-2$ & 0 & 0 & 0 & 1 \\
\hline & 2 & 0 & 1 & 0 & 0 & $2-2$ & 0 & 0 & 1 & 0 \\
\hline \multirow{4}{*}{$\begin{array}{l}\text { Two } \\
\text { move }\end{array}$} & 3 & 1 & 1 & 0 & 0 & $3-2$ & 0 & 0 & 1 & 1 \\
\hline & 4 & 1 & 0 & 1 & 0 & $4-2$ & 0 & 1 & 0 & 1 \\
\hline & 5 & 1 & 0 & 0 & 1 & & & & & \\
\hline & 6 & 0 & 1 & 1 & 0 & & & & & \\
\hline \multirow{2}{*}{$\begin{array}{l}\text { Three fingers } \\
\text { move }\end{array}$} & 7 & 1 & 1 & 1 & 0 & $7-2$ & 0 & 1 & 1 & 1 \\
\hline & 8 & 1 & 1 & 0 & 1 & $8-2$ & 1 & 0 & 1 & 1 \\
\hline $\begin{array}{ll}\text { Four } & \text { fingers } \\
\text { move } & \end{array}$ & 9 & 1 & 1 & 1 & 1 & & & & & \\
\hline
\end{tabular}




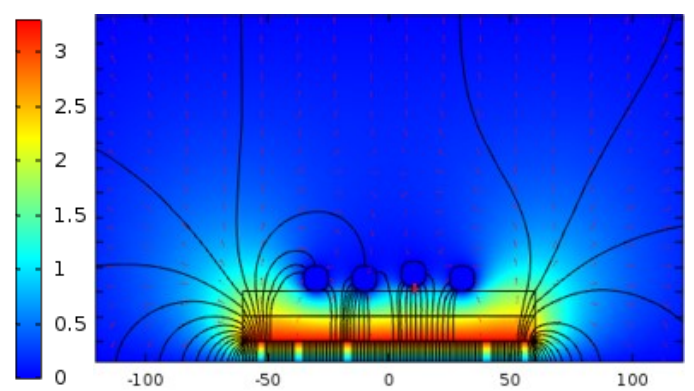

(a)

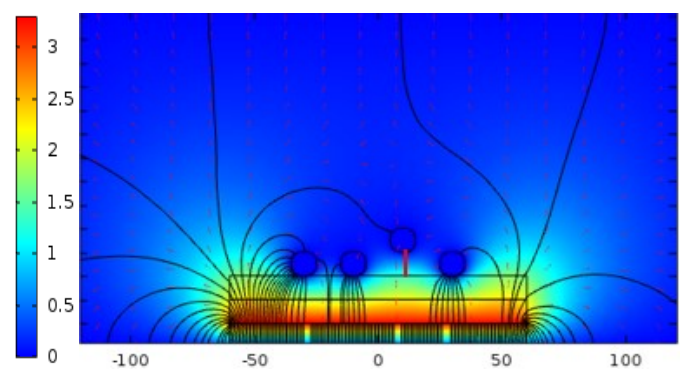

(b)

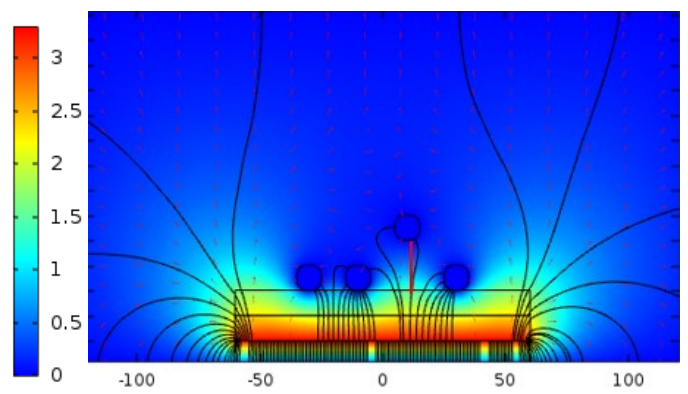

(c)

Fig. 2 Simulation model when ring finger moves (case 2-2: 0010) ((a) distance $=2 \mathrm{~mm} ;(\mathrm{b})$ distance $=10 \mathrm{~mm} ;(\mathrm{c})$ distance $=20 \mathrm{~mm})$

The plots in Fig. 3(a) with Fig. 3(b) agree with the quasistatic electrical near field theory of capacitive sensor MGC3130. Moreover, it also shows similarity that implies a symmetrical pattern of voltages of the whole electrode stack-up structure. However, the magnitude of the voltages for each specific electrode are different. The relationship of electrodes behaviour are summarized as in table 2 , which is in accordance with the symmetrical structure proposed at the start of the paper when discuss the finger motion.

TABLE 2 PAIRS OF EQUivalent ELECTRODES BeTWEen CASE2 AND CASE 2-2

\begin{tabular}{|l|l|l|l|l|}
\hline & \multicolumn{4}{|c|}{ Equivalent electrodes } \\
\hline $\begin{array}{l}\text { Case2-2 } \\
\text { (Ring finger moves) }\end{array}$ & V1 & V2 & V3 & V4 \\
\hline $\begin{array}{l}\text { Case2 } \\
\text { (Middle finger moves) }\end{array}$ & V4 & V3 & V2 & V1 \\
\hline
\end{tabular}

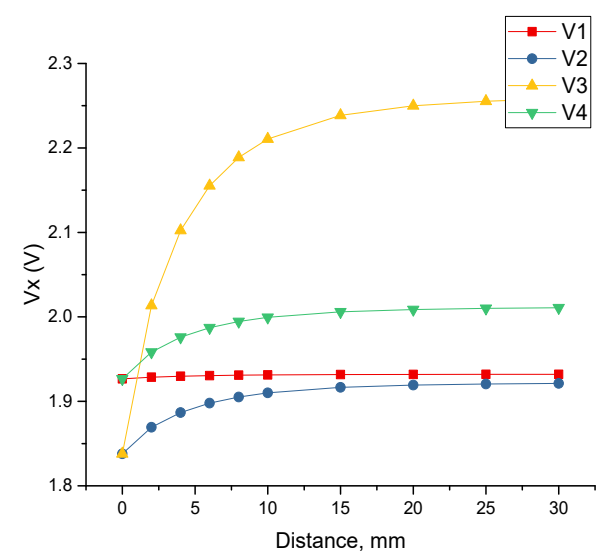

(a)

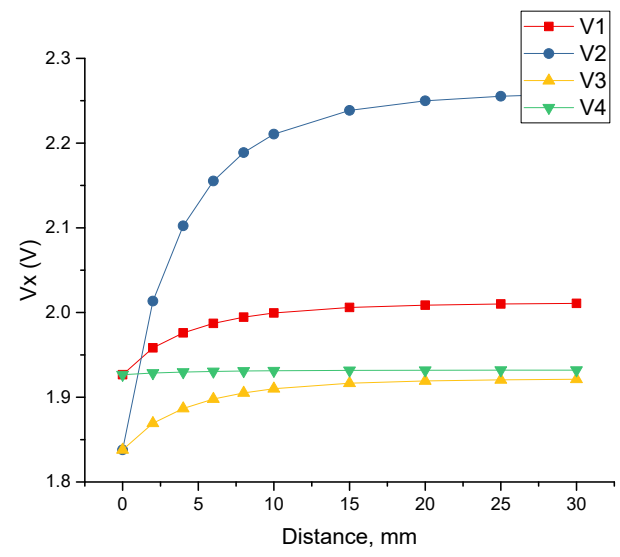

(b)

Fig. 3 Relationship between distance of fingers and the voltage of electrodes in Comsol@: (a) case2-2(0010), (b) case2 (0100)

\section{FUNCTIONAL RELATIONSHIP BETWEEN VOLTAGE AND DISTANCE}

Further to the voltage data from the Comsol ${ }^{\circledR}$ evaluation, a nonlinear regression was carried out in Matlab ${ }^{\circledR}$ for all the nine cases presented above to explore the inherent relationship between the distance of finger motion (d) and the voltage signals detected in the Rx electrodes (Vx). After attempting to fit the data with exponential, polynomial and rational functions using Excel $\AA$ and Matlab $\AA$ curve fitting tools, the data were found to fit an inverse function relation, as shown in equation (1):

$$
V x=b 1-b 2 /(d+b 3)
$$

Table 3 gives the exemplar parameters of the curve fitting results in case 2-2(0010) and case 2(0100). Here, with the listed parameters $\mathrm{b} 1, \mathrm{~b} 2$ and $\mathrm{b} 3$ and the equation (1), predicted voltage values of each $\mathrm{Vx}$ in case $2-2$ and case 2 could be calculated. 
Using case 2-2(0010), V3 as an example, Fig. 4(a) shows the real curve determined by the origin 10 points (blue ' + ') from Comsol ${ }^{\circledR}$ simulation, and the fitted curve drawn by the regression model (black '*') from Matlab®. In Fig.4 (b), the predicted values and real values were calculated and compared. Apart from the origin 10 points $(0 \mathrm{~mm}, 2 \mathrm{~mm}, 4 \mathrm{~mm}, 6 \mathrm{~mm}, 8 \mathrm{~mm}$, $10 \mathrm{~mm}, 15 \mathrm{~mm}, 20 \mathrm{~mm}, 25 \mathrm{~mm}, 30 \mathrm{~mm}$, black '*') used to conduct the regression, an extra 10 testing points $(1 \mathrm{~mm}, 3 \mathrm{~mm}$, $5 \mathrm{~mm}, 7 \mathrm{~mm}, 9 \mathrm{~mm}, 12 \mathrm{~mm}, 18 \mathrm{~mm}, 24 \mathrm{~mm}, 28 \mathrm{~mm}, 40 \mathrm{~mm}$, red '*') were also simulated to test the regression model.

TABLE 3 PARAMETERS OF NONLINEAR CURVE FITTING IN MATLABR

\begin{tabular}{|c|c|c|c|c|c|c|c|c|}
\hline \multirow{2}{*}{} & \multicolumn{4}{|c|}{ Case 2-2 } & \multicolumn{4}{c|}{ Case 2 } \\
\cline { 2 - 9 } & $\boldsymbol{b 1}$ & $\boldsymbol{b 2}$ & $\boldsymbol{b 3}$ & $\boldsymbol{R}^{\mathbf{2}}$ & $\boldsymbol{b 1}$ & $\boldsymbol{b 2}$ & $\boldsymbol{b 3}$ & $\boldsymbol{R}^{\mathbf{2}}$ \\
\hline V1 & 1.933 & 0.022 & 3.594 & 0.997 & 2.024 & 0.355 & 3.633 & 0.997 \\
\hline V2 & 1.934 & 0.351 & 3.631 & 0.997 & 2.313 & 1.453 & 3.041 & 0.997 \\
\hline V3 & 2.313 & 1.450 & 3.041 & 0.997 & 1.934 & 0.351 & 3.631 & 0.997 \\
\hline V4 & 2.024 & 0.355 & 3.632 & 0.997 & 1.933 & 0.022 & 3.595 & 0.997 \\
\hline
\end{tabular}

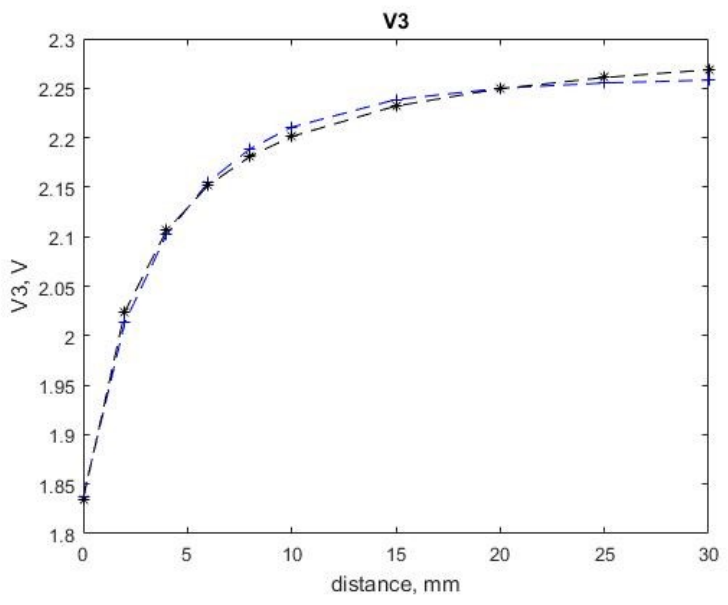

(a)

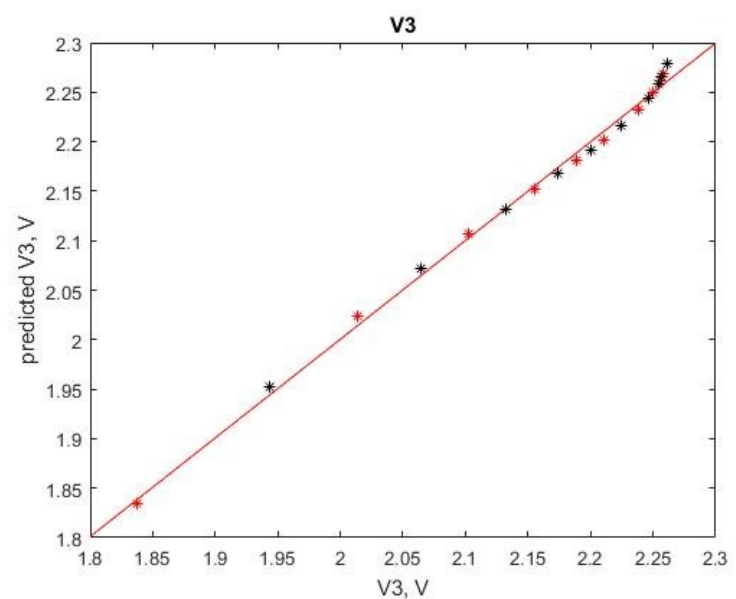

(b)

Fig. 4 Regression model in Matlab $₫$ comparing with Comsol $\AA$ simulation result (case 2-2: 0010)((a) Curve fitting result (b)) Regression model testing
Simply from the R squared values in Table 3 and the Fig. 4(b), the distance from finger motion and the voltage signals detected in Rx electrodes fits well with (1), which indicates an inverse relationship featured with three parameters, b1, b2 and b3 [21]. Moreover, there are also symmetrical features of these parameters reflected in table 3: parameters of $\mathrm{V} 1, \mathrm{~V} 2, \mathrm{~V} 3$ and V4 in case 2-2 are very close to those of V4, V3, V2 and V1 in case 2 respectively. It agrees with the pairs of equivalent electrodes proposed in table 2, which supports the symmetrical structure as well.

\section{Modified Electrode LAYER Stack-UP DESIGN}

This paper also explored a different electrode layer stack-up design in Comsol ${ }^{\circledR}$ and in Matlab for the further optimized the three-layer electrode design. The main ground electrode is denoted as GND electrode, while the smaller ones in per pair of $\mathrm{Rx}$ electrodes are denoted as 'gnd'.

A comparison was made in the two-dimensional Comsol $\AA$ simulation model, between the original design and the modified design with three extra gnd electrodes placed at the midpoints neighboring the Rx electrodes pairs, as shown in Fig. 5(a). In the Comsol ${ }^{\circledR}$ simulation model, 3.3 V A.C is applied to Tx electrode whereas both the GND electrode and the added gnd electrodes are connected to the ground potential.

Fig. 5(b) shows that when the ring finger moves away from the upper surface of the cover layer, the electric field distribution of the compared Comsol® simulation model with added gnd electrodes. Similar to the previous one, when the 'ring finger' moved away from the $\mathrm{Rx}$ electrodes, their corresponding voltage values increased as the signal to reflect this process.

The relationship between voltages of $\mathrm{Vx}$ electrodes and the distance in case 2-2 and case 2 were calculated, as presented in Fig. 6. Comparing Fig. 6(a) and Fig. 6(b), the similarity implies a symmetrical relationship of electrodes, which is in accordance with the symmetrical structure discussed in the original electrode stack-up design and also can be summarized as the table 2 .

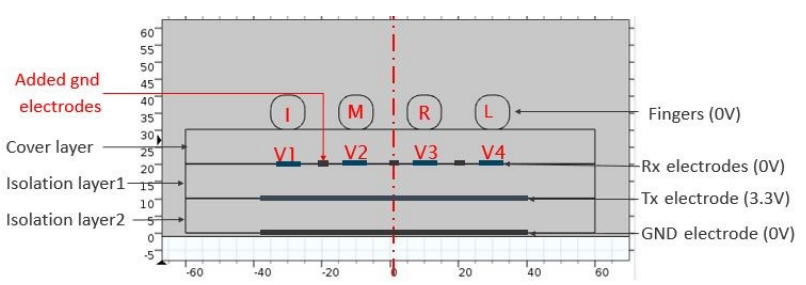

(a)

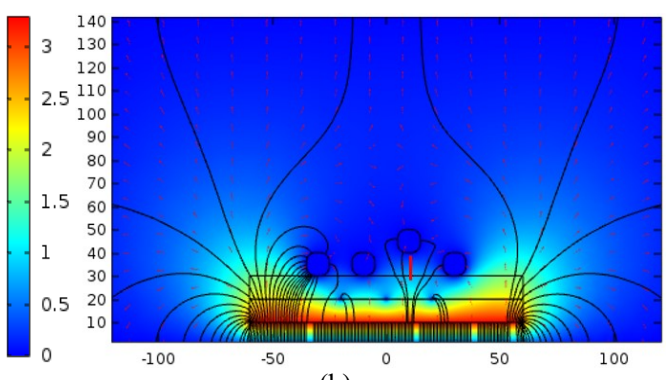

(b)

Fig. 5 Electrode stack-up design with added gnd electrodes (I: index; M: middle; R: ring; L: little): (a) simulation model; (b) Simulation model when ring finger moves (case 2-2: 0010, distance $=10 \mathrm{~mm}$ ) 


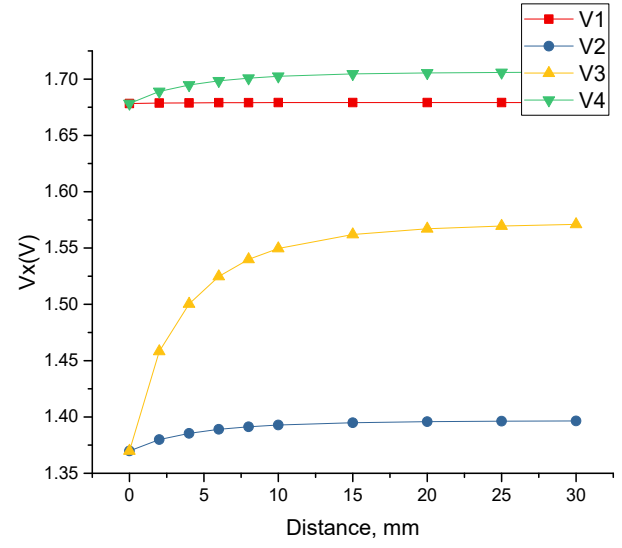

(a)

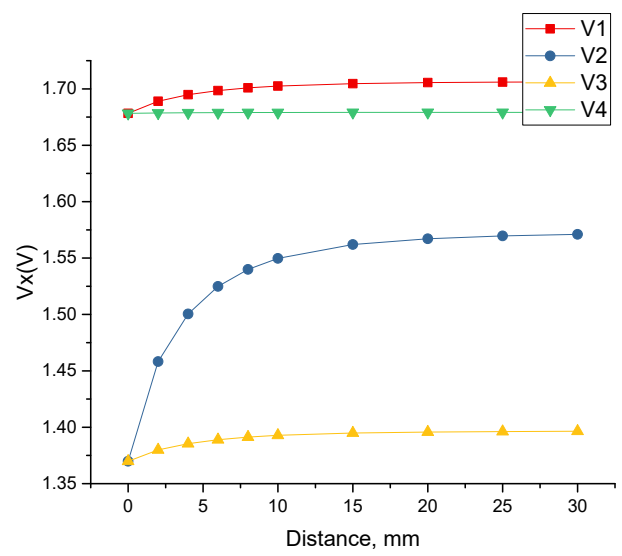

(b)

Fig. 6 Relationship between distance of fingers and the voltage of electrodes in modified Comsol® simulation: (a) case2-2(0010),(b) case $2(0100)$

In addition, the plots of case 2-2 and case 2 in Fig. 6 show similar trend comparing to the plots in the Fig. 3. Based on the essentiall from the Comsol ${ }^{\circledR}$ point evaluation, a nonlinear regression was also carried out for the best matching equation. The result to describe the relationship between the distance (d) and the voltage signals detected in the Rx electrodes $(\mathrm{Vx})$ is in compliance with (1) in this process. Table 4 gives the curve fitting result of modified electrode layer stack-up design in case 2-2(0010) and case 2(0100).

TABLE 4 CURVE FITTED PARAMETERS OF MODIFIED ELECTRODE STACK-UP DESIGN

\begin{tabular}{|c|c|c|c|c|c|c|c|c|}
\hline & \multicolumn{5}{|c|}{ Case 2-2 } & \multicolumn{4}{c|}{ Case 2 } \\
\cline { 2 - 9 } & $\boldsymbol{b 1}$ & $\boldsymbol{b 2}$ & $\boldsymbol{b 3}$ & $\boldsymbol{R}^{\mathbf{2}}$ & $\boldsymbol{b 1}$ & $\boldsymbol{b 2}$ & $\boldsymbol{b 3}$ & $\boldsymbol{R}^{2}$ \\
\hline V1 & 1.679 & 0.003 & 2.861 & 0.988 & 1.710 & 0.115 & 3.574 & 0.997 \\
\hline V2 & 1.401 & 0.112 & 3.616 & 0.997 & 1.595 & 0.631 & 2.787 & 0.997 \\
\hline V3 & 1.595 & 0.630 & 2.786 & 0.997 & 1.400 & 0.111 & 3.612 & 0.997 \\
\hline V4 & 1.710 & 0.115 & 3.569 & 0.997 & 1.679 & 0.003 & 2.775 & 0.986 \\
\hline
\end{tabular}

\section{DISCUSSION}

Comparing the fitted equation with Fig. 3, Fig. 4 and Fig. 6, the physical meaning of the three parameters in the equation can be explained:

- ' $b 1$ ' is the voltages when a finger is at a large distance where the effect of this finger can be neglected.

- ' $b 2$ ' can be regarded as a sensitive factor. It reflects the magnitude of an intruding finger to the signal read from the certain Rx electrode of an electrode layer stack-up design.

- ' $b 3$ ' is the offset value associated with the original distance of the finger to the electrode. Considering the top layer in between fingers and $\mathrm{Rx}$ electrodes will always have a finite thickness, it is reasonable to have this offset in the curve fitting equation.

With the nonlinear fitted equation to describe the inherent relationship between distance of finger motion (d) and the voltage signals detected in the $\mathrm{Rx}$ electrodes $(\mathrm{Vx})$, the performance of an electrode layer stack-up design can be described mathematically. Then, the relation identified from the simulation will be compared to experimental data to guide and optimize the final practical design. Finally, the three-layer electrode will be connected to the microcontroller for hardware and software integration as well as parameterization.

\section{CONCLUSION}

This article carried out a finite element method (FEM) simulation based on the MGC3130 electrode stack-up design using Comsol ${ }^{\circledR}$ Multiphysics. Based on the simulated data, a nonlinear regression in Matlab $\AA$ was investigated. The results agree with the quasi-static electrical near field theory and the symmetrical structure of the three-layer electrode. Moreover, the nonlinear fitted equation fits well in both the original electrode layer stack-up design and the modified electrodes design. It implies a clear inverse relation between the distance changed from finger motion and the voltage signals detected in $\mathrm{Rx}$ electrodes. Additionally, it also reflects sensitive and finite features of the electrode design, which will help to guide and optimize the future design.

\section{ACKNOWLEDGMENT}

We acknowledge University of Southampton, Hampshire, United Kingdom, the China Scholarship Council, Beijing, and Xiamen University, Fujian, China, for their support.

\section{REFERENCES}

[1]. Steven L. Wolf, Steven L. Wolf, Paul A. Thompson, Carolee J. Winstein, J. Phillip Miller, Sarah R. Blanton, et al., Nichols-Larsen et al., The EXCITE stroke trial: comparing early and delayed constraintinduced movement therapy. Stroke, 2010. 41(10): p. 2309-15.

[2]. Belén Rubio Ballester, Martina Maier, Rosa San Segundo, Victoria Castañeda Galeano, Armin Duff, Paul F.M.J. Verschure, ReinforcementInduced Movement Therapy: A novel approach for overcoming learned non-use in chronic stroke patients. in Virtual Rehabilitation Proceedings (ICVR), 2015 International Conference on. 2015. IEEE.

[3]. Lum, Peter S, Taub, Edward, Schwandt, Douglas, Automated Constraint-Induced Therapy Extension (AutoCITE) for movement 
deficits after stroke. Journal of rehabilitation Research \& Development, 2004. Volume 41 Number 3A: p. $249-258$.

[4]. Wu, C.-y., Keh-chung Lin, Ching-ping Chao, Yu-ting Chen, Constraintinduced therapy with trunk restraint for improving functional outcomes and trunk-arm control after stroke a randomized controlled trial. 2012.

[5]. Harris, N.R. and D. Sthapit. Towards a personalised rehabilitation system for post stroke treatment. in 2016 IEEE Sensors Applications Symposium (SAS). 2016. IEEE.

[6]. O'Sullivan, S., Stroke In: O'Sullivan SB and Schmitz TJ. Physical Rehabilitation. FA Davis, Philadelphia, PA, 2007: p. 706-776.

[7]. Reaz, M., M. Hussain, and F. Mohd-Yasin, Techniques of EMG signal analysis: detection, processing, classification and applications. Biological procedures online, 2006. 8(1): p. 11-35.

[8]. Zaheer, F., S.H. Roy, and C.J. De Luca, Preferred sensor sites for surface EMG signal decomposition. Physiological measurement, 2012. 33(2): p. 195.

[9]. Zhu, X. and K.F. Li. Real-time motion capture: an overview. in 2016 10th International Conference on Complex, Intelligent, and Software Intensive Systems (CISIS). 2016.

[10]. Sonkusare, J.S., Nilkanth B. Chopade, Ravindra Sor, Sunil L., A review on hand gesture recognition system. in 2015 International Conference on Computing Communication Control and Automation. 2015.

[11]. Janaki, V.M.S., S. Babu, and S.S. Sreekanth. Real time recognition of 3D gestures in mobile devices. in 2013 IEEE Recent Advances in Intelligent Computational Systems (RAICS). 2013.
[12]. Zhang, Z., Microsoft Kinect Sensor and Its Effect. IEEE MultiMedia, 2012. 19(2): p. 4-10.

[13]. Ren, Z., Junsong Yuan, Jingjing Meng, Zhengyou Zhang, Robust partbased hand gesture recognition using kinect sensor. IEEE Transactions on Multimedia, 2013. 15(5): p. 1110-1120.

[14]. Zhou, $\mathrm{H}$. and $\mathrm{H}$. Hu, Human motion tracking for rehabilitation-A survey. Biomedical Signal Processing and Control, 2008. 3(1): p. 1-18.

[15]. Covarrubias, M., M. Bordegoni, and U. Cugini, Force sensitive handles and capacitive touch sensor for driving a flexible haptic-based immersive system. Sensors, 2013. 13(10): p. 13487-13508.

[16]. Ramezani Akhmareh, A., Mihai Teodor Lazarescu, Osama Bin Tariq, Luciano Lavagno, A tagless indoor localization system based on capacitive sensing technology. Sensors, 2016. 16(9): p. 1448.

[17]. Sciberras, J., Interactive gesture controller for a motorised wheelchair. 2015, Murdoch University.

[18]. MGC3030/3130 3D Tracking and Gesture Controller Data Sheet. Available from: http://ww1.microchip.com/downloads/en/DeviceDoc/40001667C.pdf.

[19]. Logan, D.L., A first course in the finite element method. 2011: Cengage Learning.

[20]. GestIC Design Guide. Available from: http://www.microchip.com/downloads/en/DeviceDoc/40001721A.pdf.

[21]. Devore, J.L., Probability and Statistics for Engineering and the Sciences. 2011: Cengage learning. 